Article

\title{
Analysis on Abrasive Wear Rate of VGCF/Polyamide 6 Composite Fibers
}

\author{
Toshihira Irisawa $^{1)}$, Tatsuro Takamura ${ }^{1)}$, Satoshi Momozono ${ }^{2)}$, Junichi Kaneko ${ }^{3)}$ and Masatoshi Shioya ${ }^{1)^{*}}$ \\ ${ }^{1)}$ Department of Organic and Polymeric Materials, Tokyo Institute of Technology \\ 2-12-1-S8-34 Ookayama, Meguro-ku, Tokyo 152-8552, Japan \\ ${ }^{2)}$ Department of Mechanical and Aerospace Engineering, Tokyo Institute of Technology \\ 2-12-1-I1-39 Ookayama, Meguro-ku, Tokyo 152-8550, Japan \\ ${ }^{3)}$ Nippon Felt Co., Ltd. \\ 88 Haramamuro, Konosu-shi, Saitama 365-0043, Japan \\ ${ }^{*}$ Corresponding author: shioya.m.aa@m.titech.ac.jp
}

( Manuscript received 16 November 2010; accepted 9 February 2011; published 31 March 2011 )

\begin{abstract}
The tribological properties under abrasive wear conditions, the tensile properties, the thermal properties and the structure have been investigated on the polyamide 6 (PA6) fibers dispersed with vapor-grown carbon fibers (VGCF's) in order to analyze the effects of dispersing VGCF's on the wear resistance of PA6 fibers. The wear resistance of the fibers have been determined using a method previously proposed by the present authors. The coefficient of friction decreased by dispersing a small amount of VGCF's but increased with increasing VGCF fraction at large VGCF fractions. The wear resistance increased with increasing VGCF fraction. Under the experimental conditions adopted in this study, the dispersion of VGCF's more effectively increased the wear resistance of PA6 fibers than the heat treatment. These experimental results have been analyzed based on a model with which the coefficient of friction $(\mu)$ and the wear rate $\left(W_{s}\right)$ have been related to the microhardness $(H)$, the resistance against sliding $\left(\sigma_{s}\right)$, the fracture probability $(f)$ the shape factor $(g)$ and a constant $(k)$ as $\mu=g \sigma_{s} /(k H)$ and $W_{s}=g f /(k H)$.
\end{abstract}

Keywords: abrasive wear, wear rate, coefficient of friction, polyamide 6, VGCF, fiber, composite

\section{Introduction}

Polymeric materials are used under various tribological conditions in place of metals and ceramics on the occasion where characteristic properties of polymeric materials such as flexibility and good processability are of importance ${ }^{1,2)}$. An example is the fiber used for paper-making belts which are exposed to severe abrasive conditions when they are in contact with other parts of the paper-making machine. In addition, this fiber needs to sustain large tension to drive the belts at a high speed. Although polyamide 6 (PA6) fibers are currently used for this purpose, it is desired to increase the resistance of the fibers against abrasive wear in order to increase the driving speed of the belts. The tensile modulus and strength of PA6 fibers can be increased by heat treatment and drawing as a result of the increases in the crystallinity and the molecular orientation $^{3)}$. The increases in the tensile modulus and strength are favorable to increase the wear resistance. Our previous study ${ }^{4,5)}$, however, showed that the wear resistance of PA6 fibers was decreased by heat treatment and drawing. This result has been attributed to the decreases in the elongation at break and the shear strength against the force applied on the surface of the fiber, due to the increases in the crystallinity and the anisotropy of the structure, respectively.

It is expected that the wear resistance of PA6 fibers can be increased effectively by dispersing fillers. The effects of dispersing fillers on the wear resistance of polymers in the form of films and bulk specimens have been studied by many researchers. Based on the consideration that the wear resistance is in proportion to the product of the tensile strength and the elongation at break $^{6-8)}$, the wear resistance of polymers dispersed with fillers has often been analyzed using the plots of wear volume against the product of the tensile strength and the elongation at break (Rantner-Lancaster plots) ${ }^{9-12)}$. Based on the model analysis by Lhymn showing that the wear resistance of polymer composites is in proportional to the tensile modulus or the hardness, the wear resistance of polymers dispersed with fillers has also been analyzed in relation with the tensile modulus and the hardness ${ }^{13)}$. The increase in the wear resistance of polymers by dispersing fillers has been attributed mainly to two factors: one being the changes in the polymer structure developed 
during processing ${ }^{14,15)}$ and the other being the contribution of the mechanical properties of the fillers itself such as the high tensile strength, high tensile modulus and high hardness ${ }^{16-18)}$.

The importance of the large aspect ratio of the fillers to increase wear resistance has been recognized with the fillers such as carbon fibers and glass fibers ${ }^{19-22)}$. This suggests that carbon nanofibers (CNF) having a large aspect ratio and a high tensile modulus are desirable fillers to increase the wear resistance of the polymer. A number of studies have been carried out on the tensile modulus and strength, the fracture toughness and the fatigue of the CNF dispersed polymers ${ }^{23-26)}$. As to the frictional properties and wear resistance, it has been shown that dispersion of a small amount of CNF's into polymers has large influences on these properties ${ }^{27-30)}$. The increase in the wear resistance of polymers by dispersing CNF's has been attributed to the bridging effect, reinforcement of the transfer films formed between the polymer and the counterface, self-lubrication, and the high thermal conductivity which contributes to release frictional heat ${ }^{30)}$. Most of these studies focused on the adhesive wear of the films and the bulk specimens. It is worth while to study the influences of dispersing CNF's into polymers on the abrasive wear resistance of the fibers. It is considered that the difference in the molecular orientation between the films and the fibers, and the difference in the sizes of scars produced under the adhesive and abrasive wear will have a large difference in the effects of dispersing fillers on the wear resistance of polymers.

In this study, the abrasive wear resistance of PA6 fibers dispersed with vapor-grown carbon fibers (VGCF's) has been investigated in relation to other mechanical properties and the structure of the fibers. For determining wear rate of fibers, a method proposed by the present authors has been used ${ }^{5)}$.

\section{Experimental}

\subsection{Materials}

The extrusion and blow application grade PA6 (Unitika M1040) with the glass transition temperature at $48.5^{\circ} \mathrm{C}$ and the melting temperature at $223^{\circ} \mathrm{C}$ was used as the matrix polymer. VGCF's (Showa Denko, VGCF-S) about $150 \mathrm{~nm}$ in diameter and several micrometers in length with the aspect ratio of about $10-$ 500 were used as the fillers.

The VGCF/PA6 composite fibers were prepared as follows: The compounds of PA6 containing 5 mass $\%$ VGCF's and the pellets of neat PA6 were dried at $80{ }^{\circ} \mathrm{C}$ for $24 \mathrm{~h}$ in a vacuum oven and blended so that the mass fraction of VGCF's in the blend varied between 0 and 5 mass $\%$. The blends were hot-pressed at $250{ }^{\circ} \mathrm{C}$ into a film and immediately quenched by placing the film between two steel plates cooled with water at room temperature. The film was cut into small pieces and stirred in order to enhance dispersion of VGCF's in PA6. Then, the pieces were hot-pressed into the film again with the same procedure as shown above. This film forming-cutting cycles were repeated and the films obtained at the third hot-pressing were used for melt spinning and measurements. The same process history was imposed on the neat PA6. The fibers were melt spun using a single hole spinneret with a diameter of $1.5 \mathrm{~mm}$ at a throughput rate of $1 \mathrm{~g} \mathrm{~min}^{-1}$, a take-up velocity of $2 \mathrm{~m} \mathrm{~min}^{-1}$, and an extrusion temperature of $250{ }^{\circ} \mathrm{C}$. The fiber diameter was about $0.5 \mathrm{~mm}$ and the film thickness was about $0.3 \mathrm{~mm}$. Some of the fibers and the films were heat treated under constant length at a temperature of $200{ }^{\circ} \mathrm{C}$ for $15 \mathrm{~min}$ in the air.

\subsection{Crystallite structure}

Wide-angle X-ray diffraction (WAXD) was measured on the aligned fibers using a diffractometer (Rigaku, CN4036A2), a position-sensitive proportional counter (PSPC), a proportional counter and a Ni filtered $\mathrm{CuK} \alpha$ radiation. The equatorial diffraction profiles were measured with the PSPC in a manner that the incident beam, the specimen and the diffracted beam toward the center of the detecting window of the PSPC were in the symmetric transmission geometry. The azimuthal diffraction profiles were measured with the proportional counter by rotating the specimen keeping the symmetric transmission geometry.

\subsection{Thermal properties}

A differential thermal analysis (DTA) of the fibers was carried out using a TG-DTA apparatus (Rigaku, TG8120). Before the measurements, the fibers were dried at $80^{\circ} \mathrm{C}$ for $24 \mathrm{~h}$ in a vacuum oven to eliminate absorbed water. The DTA measurements were carried out on the chopped fibers of about $5 \mathrm{mg}$ using an aluminum cell under the nitrogen gas flow at a heating and cooling rate of $10{ }^{\circ} \mathrm{C} \mathrm{min}$. The DTA curve of an $\mathrm{Al}_{2} \mathrm{O}_{3}$ standard was also measured in order to calculate the enthalpy change of the fibers from the area of the DTA curve.

The melting temperature $\left(T_{m}\right)$ and the crystallization temperature $\left(T_{c}\right)$ were determined as the peak temperatures of the DTA curves measured during the first heating and the first cooling processes, respectively. The degree of crystallinity of PA6 $\left(\chi_{c}\right)$ was calculated according to the equation ${ }^{31)}$

$$
\chi_{c}=\frac{\Delta H_{m}}{\Delta H_{m, o}(1-m)} \times 100
$$

where $\Delta H_{m, o}$ is the specific melting heat of PA6 crystal, $\Delta H_{m}$ is the specific melting heat of the VGFC/PA6 composite fiber and $m$ is the mass fraction of VGCF's in the composite fiber. The values of $\Delta H_{m, o}$ for the $\alpha$ and $\gamma$ phases are 241 and $239 \mathrm{~J} \mathrm{~g}^{-131,32)}$, respectively, and the average value, $240 \mathrm{~J} \mathrm{~g}^{-1}$ was used for $\Delta H_{m, o}$ in this study.

\subsection{Tensile properties}

Tensile tests were carried out on the single fibers using a tensile testing machine (Orientec, RTC-1350A). 


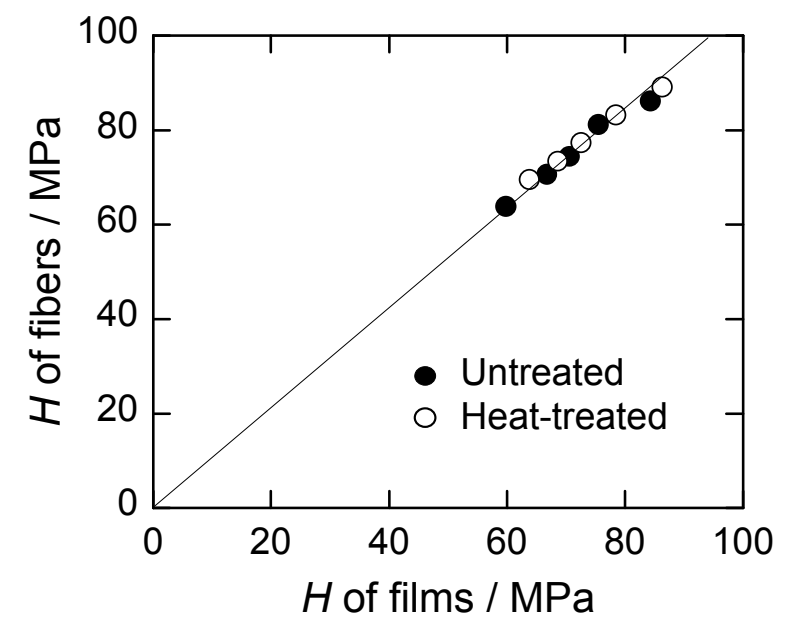

Fig. $1 H$ of fibers versus $H$ of films for untreated and heat-treated VGCF/PA6 composites fibers and films with various mass fractions of VGCF's

The gage length was $30 \mathrm{~mm}$ and the crosshead speed was $30 \mathrm{~mm} \mathrm{~min}^{-1}$. The tensile modulus, the tensile strength and the work of fracture were determined from the initial slope of the stress-strain curve, the maximum load attained at the fiber break and the area under the stress-strain curve, respectively. For each specimen, 25 determinations were performed and the average value will be shown.

\subsection{Microhardness}

The microhardness measurements were carried out on the films and the fibers using a Vicker's microhardness tester (SHIMADZU, ENT-150). The measurements were carried out by applying the normal load of $0.5 \mathrm{~N}$ to an indenter for the indentation time of $15 \mathrm{~s}$. The diagonal sizes of the scars after indentation were in a range from 50 to $100 \mu \mathrm{m}$. The microhardness $(H)$ was calculated by dividing the applied load with the surface area of the scar of the indentation. For each specimen, 10 determinations were performed and the average value will be shown.

Fig. 1 compares $H$ of the fibers with that of the films for all the neat and the composite fibers and films used in this study. Although the films and the fibers show almost the same values of $H$, the fibers show slightly higher values than the films. This is because the fibers presented smaller scars than the films due to the curvature of the fiber surface. Therefore, the value of $H$ for the films will be used as the microhardness of the materials.

\subsection{Coefficient of friction and wear rate}

The fiber wear tests were performed by wearing one side of a single fiber using a fiber wearing apparatus as shown in Fig. 2. This apparatus consisted of a rotating drum, an abrasive paper covering the drum, a load cell to measure the tension of the fiber, a weight to apply tension to the fiber, and the nozzle and tube for pouring water. The drum was $100 \mathrm{~mm}$ in diameter and rotated at

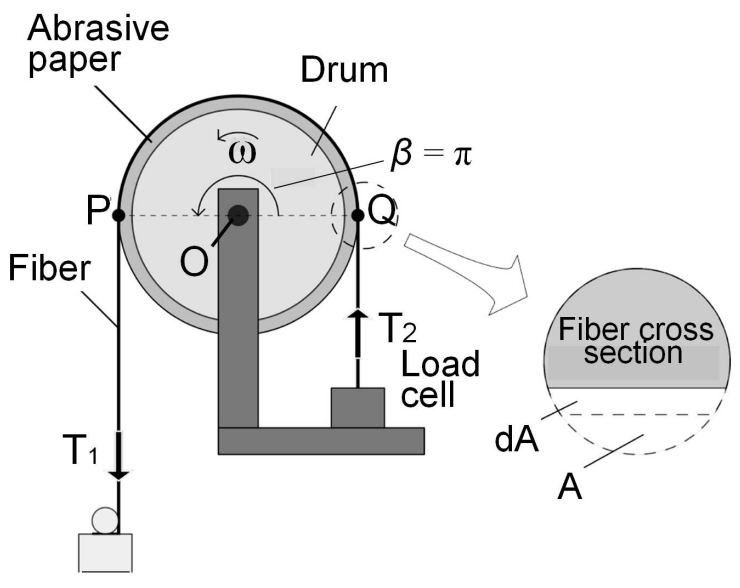

Fig. 2 Schematic illustrations of fiber wearing apparatus and fiber cross section at worn portion

a speed of $150 \mathrm{rpm}$. The average sizes of the $\mathrm{SiC}$ abrasive particles on the abrasive paper were 50 and 14 $\mu \mathrm{m}$, which are denoted as \#320 and \#800 (ISO 6344-1 and 6344-3), respectively. One end of the single fiber specimen was fixed to the load cell and the weight of 40 $\mathrm{g}$ was connected to the other end of the fiber. Then, the fiber was hung on the rotating drum for $60 \mathrm{~s}$ to wear the side of the fiber. During the wear tests, water at $20{ }^{\circ} \mathrm{C}$ was poured on the abrasive paper in order to keep the abrasive paper clean and remove the frictional heat. Fig. 3 shows the SEM images of the \#320 and \#800 abrasive paper before and after the wear tests of the untreated neat PA6 fibers under the tension of $0.39 \mathrm{~N}$ for $60 \mathrm{~s}$. Although a few fine wear debris can be seen for \#800 abrasive paper, most of the SiC particles are clearly seen on both abrasive paper. Neither the transfer film nor the

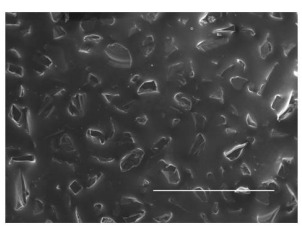

(a)

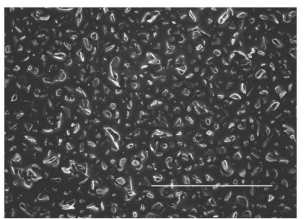

(c)

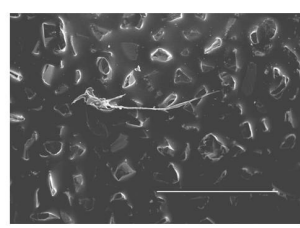

(b)

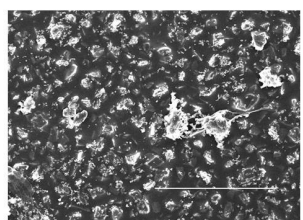

(d)
$300 \mu \mathrm{m}$
Fig. 3 SEM images of ( $a$ and b) \#320 and (c and d) \#800 abrasive paper (a and c) before and (b and d) after wear tests on PA6 fibers under tension of $0.39 \mathrm{~N}$ for $60 \mathrm{~s}$ 
adhesion of the polymer by the melting due to frictional heat can be seen. This indicates that the water poured on the surface of the abrasive paper effectively cleaned the surface of the abrasive paper and removed the frictional heat.

After the wear test, the fiber was cut at the most worn cross section and the cross-section area lost by wear was measured with a profile projector (Nikon, 6CT2). The most worn portion of the fiber was always located within $3 \mathrm{~mm}$ from the point Q in Fig. 2.

The specific wear rate $\left(W_{s}\right)$ is defined as

$$
W_{s}=\frac{V}{F L}
$$

where $V$ is the volume lost by wear, $F$ is the normal load applied on the wear surface and $L$ is the sliding distance $^{33)}$. With the wear tests on bulk and film specimens using a pin-on-disk ${ }^{34)}$ and a block-on-ring ${ }^{35)}$ apparatus, the values of $V$ and $F$ can be directly measured. With the wear tests on fibers, however, direct measurements of these values are not easy. Using an analysis method proposed by the present authors ${ }^{5)}$, the wear rate of fibers can be determined as

$$
W_{s}=\frac{1}{\omega T} \frac{d A}{d t}
$$

where $t$ is the wear time, $A$ is the fiber cross-section area lost by wear, $\omega$ is the angular speed of the rotating drum and $T$ is the fiber tension. The average specific wear rate $\left(<W_{s}>\right.$ ) during the wear test for the duration $t$ can be obtained as

$$
\left\langle W_{s}\right\rangle \equiv \frac{1}{t} \int_{0}^{t} W_{s} d t=\frac{A}{\omega T t}
$$

provided that the changes in $\omega$ and $T$ during the wear test are small.

The coefficient of friction $(\mu)$ between the fiber and the abrasive paper during the wear test can be determined $\operatorname{as}^{36,37)}$

$$
\mu=\frac{1}{\beta} \ln \left(\frac{T_{2}}{T_{1}}\right)
$$

where $\beta$ is the angle between the radii OP and OQ shown in Fig. 2 and $T_{1}$ and $T_{2}$ are the fiber tension at points $P$ and $\mathrm{Q}$, respectively. In this study, $\beta$ was $\pi, T_{1}$ was $0.39 \mathrm{~N}$ and $T_{2}$ which is equal to $T$ in equation (4) was directly measured with the load cell.

\section{Results and discussion}

\subsection{Structure and thermal properties}

The equatorial WAXD profiles of the composite fibers are shown Fig. 4. The crystallization temperature, the melting temperature and the crystallinity of PA6 in the composite fibers determined with DTA are shown in Table 1.
The PA6 crystal exhibits polymorphism and forms $\alpha$, $\beta, \gamma$ and pseudohexagonal phases ${ }^{32,38,39)}$. Thermodynamically stable phases are the $\alpha$ and the $\gamma$ phases in which polymer chains take a fully extended

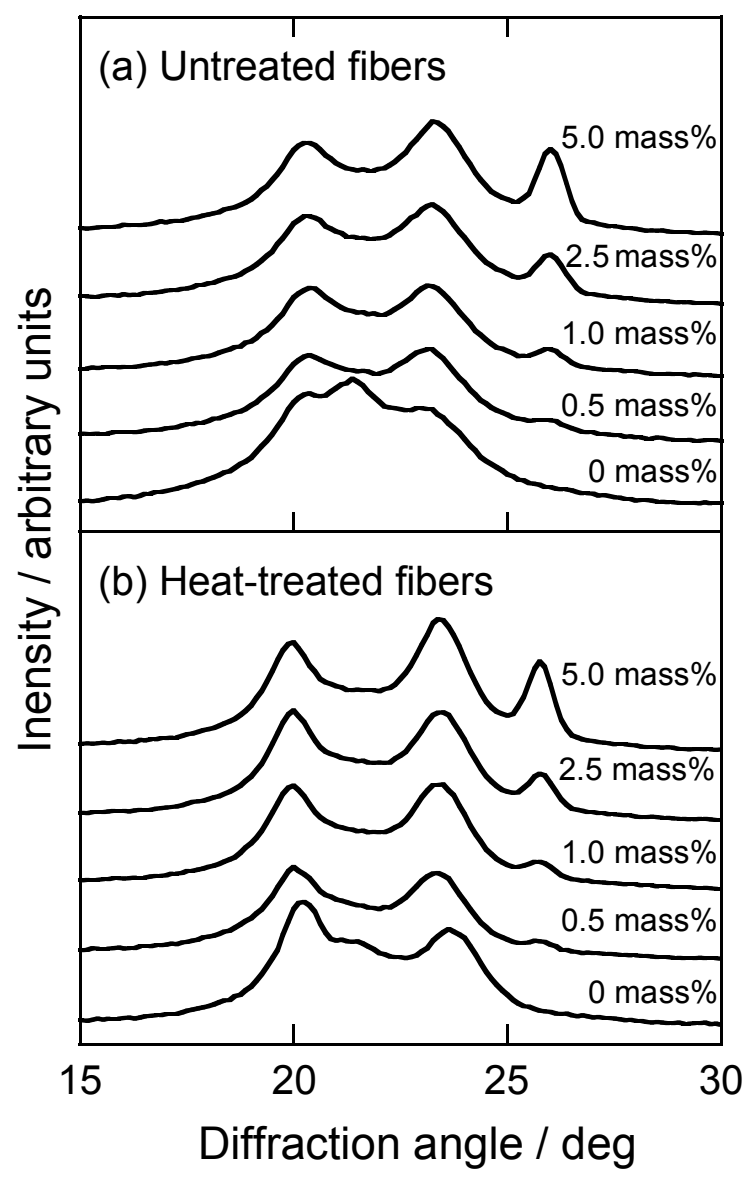

Fig. 4 Equatorial WAXD profiles of (a) untreated and (b) heat-treated VGCF/PA6 composite fibers with various mass fractions of VGCF's shown in the figure. Profiles have been shifted vertically to prevent overlap

Table 1 Crystallization temperature $\left(T_{c}\right)$, melting temperature $\left(T_{m}\right)$ and crystallinity $\left(\chi_{c}\right)$ of

\begin{tabular}{|c|c|c|c|c|c|c|}
\hline \multirow{2}{*}{$\begin{array}{l}\text { Fraction } \\
\text { of VGCF's } \\
(\text { mass } \%)\end{array}$} & \multicolumn{3}{|c|}{ Untreated fibers } & \multicolumn{3}{|c|}{ Heat-treated fibers } \\
\hline & $\begin{array}{c}T_{c} \\
\left({ }^{\circ} \mathrm{C}\right)\end{array}$ & $\begin{array}{c}T_{m} \\
\left({ }^{\circ} \mathrm{C}\right)\end{array}$ & $\begin{array}{l}\chi_{c} \\
(\%)\end{array}$ & $\begin{array}{c}T_{c} \\
\left({ }^{\circ} \mathrm{C}\right)\end{array}$ & $\begin{array}{c}T_{m} \\
\left({ }^{\circ} \mathrm{C}\right)\end{array}$ & $\begin{array}{l}\chi_{c} \\
(\%)\end{array}$ \\
\hline 0 & 188.1 & 217.8 & 24.9 & 189.3 & 218.9 & 33.3 \\
\hline 0.5 & 191.6 & 218.5 & 23.2 & 192.1 & 218.8 & 28.5 \\
\hline 1.0 & 191.2 & 218.3 & 23.4 & 191.3 & 219.2 & 30.2 \\
\hline 2.5 & 191.8 & 219.2 & 22.2 & 191.0 & 218.9 & 33.4 \\
\hline 5.0 & 194.6 & 219.3 & 23.8 & 192.7 & 219.0 & 27.1 \\
\hline
\end{tabular}
untreated and heat-treated VGCF/PA6 composite fibers with various mass fractions of VGCF's 


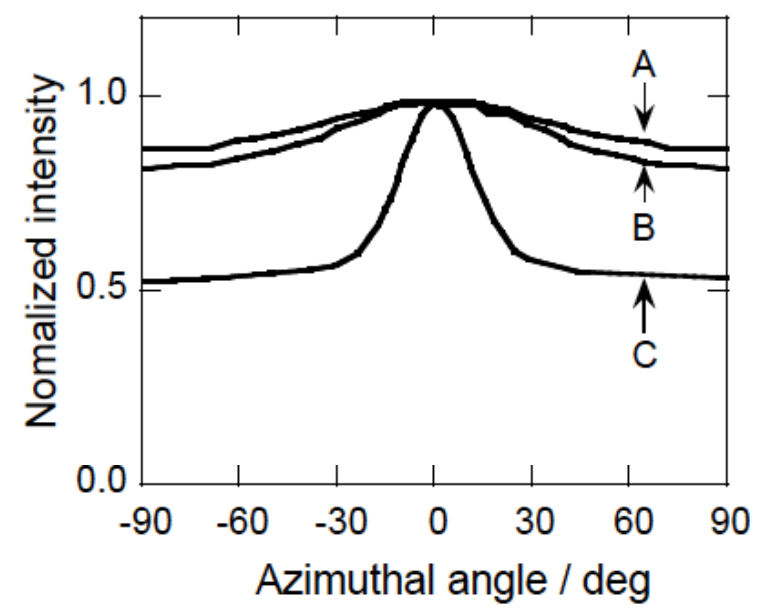

Fig. 5 Azimuthal WAXD profiles of 200 diffractions from PA6 $\alpha$ phase for (A) untreated PA6 fiber and (B) untreated 5 mass $\%$ VGCF/PA6 composite fiber and that of 002 diffraction from VGCF's for (C) untreated $5 \mathrm{mass} \% \quad \mathrm{VGCF} / \mathrm{PA} 6$ composite fiber. Azimuthal angle is $0^{\circ}$ at equatorial direction. Profiles have been normalized with the maximum intensities

planar zigzag conformation and a twisted conformation, respectively. Thermodynamically instable phases are the $\beta$ and the pseudohexagonal phases which are easily transformed into the $\alpha$ phase by the heat treatment. The $\gamma$ and the pseudohexagonal phases produce similar WAXD peaks but are distinguishable based on the thermal stability $^{39)}$.

In the diffraction profiles shown in Fig. 4 , the peaks at the scattering angles $(2 \theta)$ of $20.5^{\circ}$ and $24.0^{\circ}$ are respectively the 200 diffraction and the overlap of the 002 and the 202 diffractions from the $\alpha$ phase. In the diffraction profile of the untreated neat PA6 fiber, the peak at $2 \theta$ of $21^{\circ}-22^{\circ}$ is the 001 diffraction from the pseudohexagonal phase since this diffraction was not produced after the heat treatment and the diffraction from the $\alpha$ phase was produced alternatively. Fig. 4 shows that the pseudohexagonal phase has been developed in the untreated neat PA6 fibers while the $\alpha$ phase has been developed in the heat-treated neat PA6 fibers and the composite fibers. It is known, therefore, that VGCF's work as the nucleation agent to form the $\alpha$ phase during the cooling process from the molten state. Reflecting the nucleation effect, the crystallization temperature of PA6 under the existence of VGCF's is higher than that of the neat PA6 as shown in Table 1. By dispersing VGCF's, the melting temperature is slightly increased while the crystallinity is not increased. By the heat treatment of the composite fibers, the crystallinity is markedly increased while the melting temperature is not changed. The nucleation effect to form the PA6 $\alpha$ phase has also been reported with multi-walled carbon nanotubes ${ }^{24,31)}$.

In the diffraction profiles of the composite fibers shown in Fig. 4, the peak at $2 \theta$ of $26.0^{\circ}$ is the 002 diffraction of the carbon layer stacks in VGCF's. The intensity of this peak increases as the VGCF content increases.

Fig. 5 shows azimuthal WAXD profiles of the 200 diffractions of the PA6 $\alpha$ phase at $2 \theta$ of $20.5^{\circ}$ for the untreated neat PA6 fiber and the untreated 5 mass $\%$ VGCF/PA6 composite fiber and the profile of the 002 diffraction of VGCF's at $2 \theta$ of $26.0^{\circ}$ for the untreated 5 mass $\%$ VGCF/PA6 composite fiber. The background intensity from PA6 has not been removed from the profile of VGCF's shown in this figure. With the untreated 5 mass\% VGCF/PA6 composite fiber, the orientation of the VGCF's is much higher than that of the PA6 $\alpha$ phase. The low orientation of the PA6 $\alpha$ phase results from the small spinning stress during the melt spinning carried out at a low take-up velocity. On the other hand, a large aspect ratio of VGCF's helps them to align in parallel to the fiber axis. The orientation of VGCF's does not influence the orientation of the PA6 $\alpha$ phase as is known by comparing the azimuthal diffraction profiles of the neat and the composite fibers.

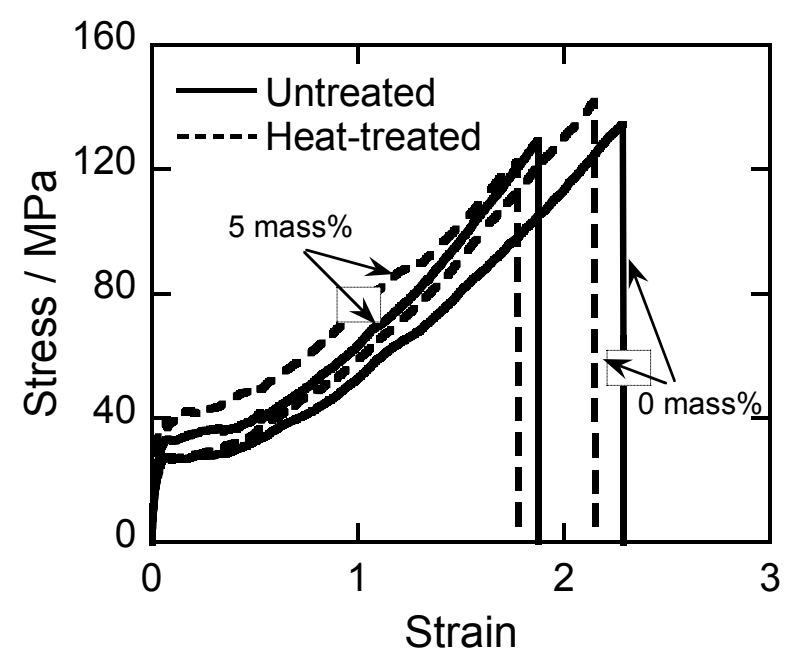

Fig. 6 Tensile stress-strain curves for untreated and heat-treated PA6 fibers and 5 mass $\%$ VGCF/PA6 composite fibers

\subsection{Tensile properties}

Typical tensile stress-strain curves of the neat PA6 fibers and the composite fibers are shown in Fig. 6. The tensile modulus, tensile yield stress, tensile strength, elongation at break and work of fracture for the composite fibers are plotted against the VGCF fraction in Fig. 7(a) to (e). By the heat treatment, the tensile modulus and strength increase but the elongation at break decreases irrespective of the VGCF fraction. It has been reported that the tensile modulus and strength of PA6 fibers are increased by the change of the crystal form from the pseudohexagonal phase to the $\alpha$ phase ${ }^{39-41)}$, the increase in the crystallinity and the increase in the crystallite orientation but significantly 

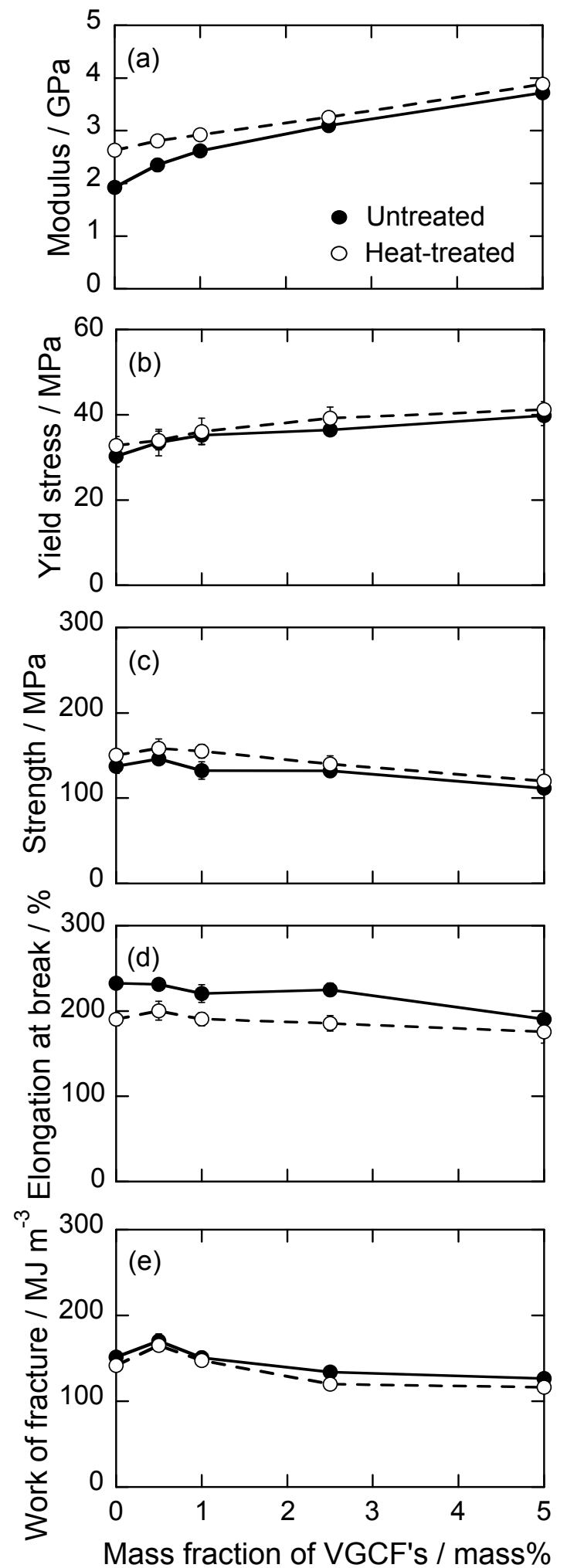

Fig. 7 (a) Tensile modulus, (b) tensile yield stress, (c) tensile strength, (d) elongation at break and (e) work of fracture versus mass fraction of VGCF's for untreated and heat-treated VGCF/PA6 composite fibers decreased by the absorption of water ${ }^{42)}$. The increase in the crystallinity by the heat treatment at any VGCF fractions is considered to be the cause of the increases in the tensile modulus and strength, and the decrease in the elongation at break. The transformation of the crystal form from the pseudohexagonal phase to the $\alpha$ phase is considered to give an additional contribution to the increase in the tensile modulus and strength by the heat treatment of the neat PA6 fiber.

By dispersing VGCF's, the tensile modulus increases but the tensile strength and the elongation at break decrease for both the untreated and the heat-treated composite fibers at the VGCF fractions larger than 0.5 mass\%. The increase in the tensile modulus with increasing VGCF fraction is considered to be the contribution of the high tensile modulus of VGCF's having a large aspect ratio and a preferential orientation in the fiber axis direction. There may be an additional contribution from the change of the crystal form from the pseudohexagonal phase to the $\alpha$ phase with the untreated fibers at a low VGCF fraction. The increase in the tensile modulus by the dispersion of VGCF's indicates that the stress applied to the composite fibers can be transferred effectively to the VGCF's through filler/matrix interfacial bonding. The decrease in the tensile strength by the dispersion of VGCF's suggests that the filler/matrix interfaces are debonded during the composite fibers are stretched beyond the elastic region up to the strain at fiber break and the debonded VGCF's work as the defects.

\subsection{Tribological properties and microhardness}

Fig. 8 shows the SEM images of the wear surfaces of the composite fibers worn with \#320 and \#800 abrasive paper under the tension of $0.39 \mathrm{~N}$ for $60 \mathrm{~s}$. The surfaces worn with \#800 abrasive paper present smoother and shallower scars as compared with the surfaces worn with \#320 abrasive paper. The composite fibers and the heat-treated neat PA6 fibers present smoother wear surfaces as compared with the untreated neat PA6 fibers. The VGCF's lying on the wear surface, bridging the banks of scars can be observed in Fig. 9.

The neat PA6 fibers and the composite fibers used in this study showed the variations of the coefficient of friction $(\mu)$ and the wear rate $\left(W_{s}\right)$ with the wear time similar to those reported in a previous paper ${ }^{5)}$. The value of $\mu$ reached almost a constant value in less than $10 \mathrm{~s}$ after showing initial rise and overshoot. The values of $W_{s}$ also reached a steady value after showing initial anomalous wear. The anomalous wear of the PA6 fibers at the very beginning of the wear tests has been attributed to the change of the surface roughness from that of the unworn surface to that of the slightly worn surface, and not to the formation of the transfer films nor the wear debris.

The microhardness $(H)$ and the average values of $\mu$ and $W_{s}$ during the initial $60 \mathrm{~s}$ of the wear tests under the tension of $0.39 \mathrm{~N}$ for the composite fibers are plotted against the VGCF fraction in Fig. 10(a) to (c). In this 


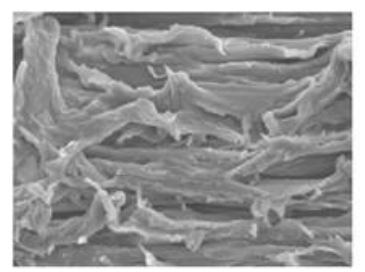

(a)

$\overline{10 \mu m}$

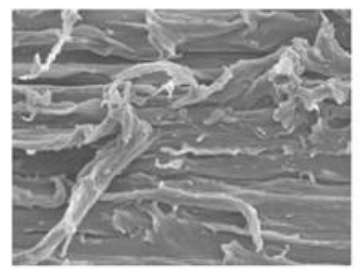

(c)
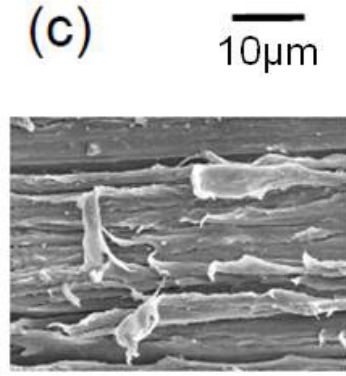

(e)
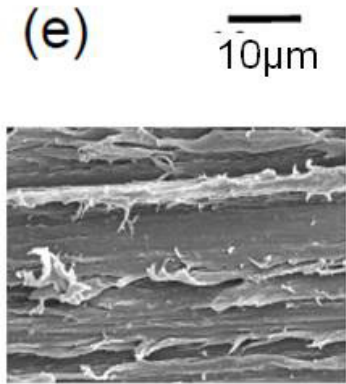

(g)

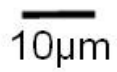

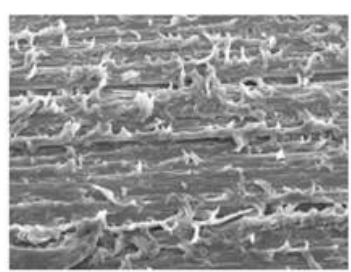

(b)

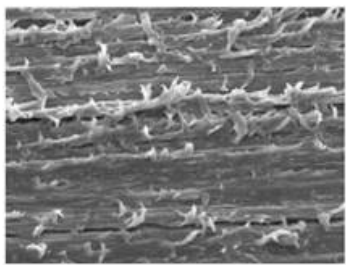

(d)
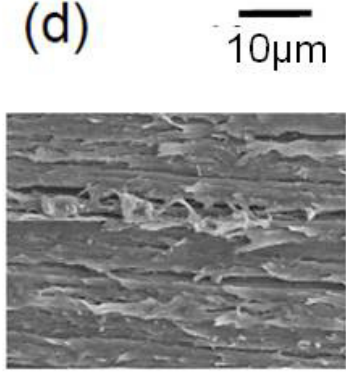

(f)
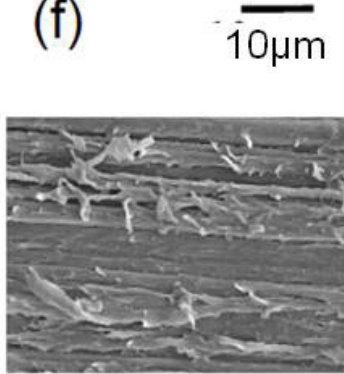

(h)

$\overline{10 \mu \mathrm{m}}$

Fig. 8 SEM images of wear surfaces for (a-d) neat PA6 fibers and (e-h) 5 mass $\%$ VGCF/PA6 composite fibers worn with (a, c, e and g) \#320 and (b, d, f and h) \#800 abrasive paper under tension of $0.39 \mathrm{~N}$ for 60 s. Wear surfaces of (a, b, e and f) untreated and (c, d, g and h) heat-treated fibers are presented

figure, the values of $H$ and $\left\langle W_{s}>\right.$ are shown in the units of $\mathrm{MPa}$ for convenience of comparison with other mechanical properties. The units often used for these values in the literature are $\mathrm{kgf} \mathrm{m} \mathrm{m}^{-2}$ for the vickers hardness and $\mathrm{mm}^{2} \mathrm{~N}^{-1}$ for the wear rate. These units can be converted as $1 \mathrm{MPa}=0.102 \mathrm{kgf} \mathrm{m}^{-2}$ and $1 \mathrm{~Pa}^{-1}=1.0 \times$ $10^{6} \mathrm{~mm}^{2} \mathrm{~N}^{-1}$. It is found in Fig. 10 that $H$ increases and $<$ $W_{s}>$ decreases as the VGCF fraction increases. The value of $\mu$ decreases by the dispersion of $0.5-1$ mass $\%$ VGCF's while it increases by the dispersion of a larger

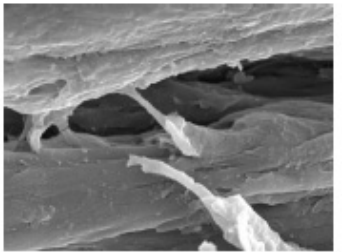

(a)

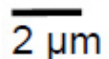

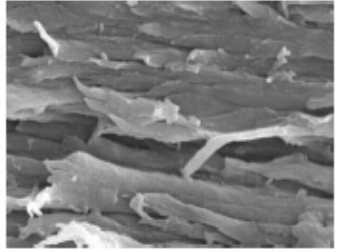

(b)

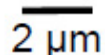

Fig. 9 SEM images of the VGCF's on worn surfaces of (a and b) untreated 5 mass $\%$ VGCF/PA6 composite fiber
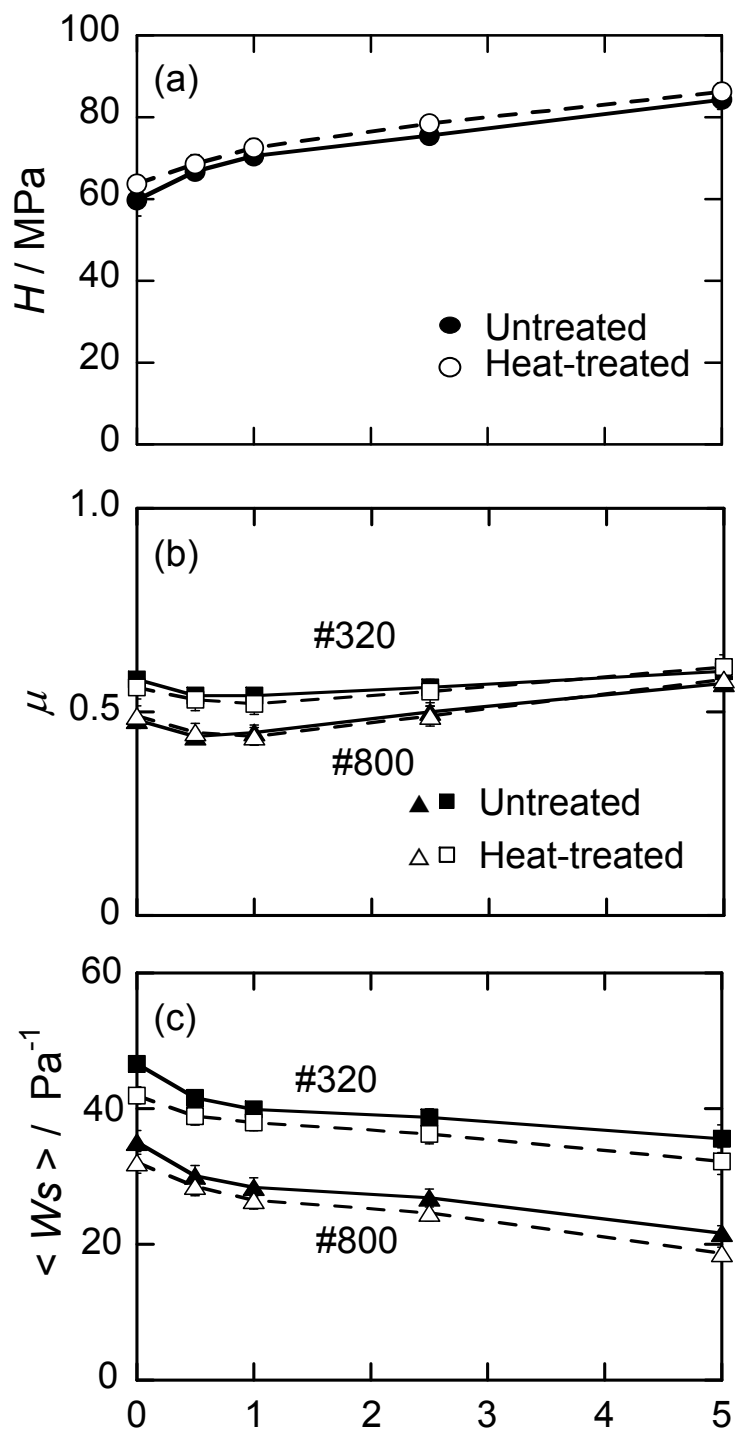

Mass fraction of VGCF's / mass $\%$

Fig. 10 (a) $H$, (b) $\mu$ and (c) $<W_{s}>$ versus mass fraction of VGCF's for untreated and heat-treated VGCF/PA6 composite fibers. Fibers were worn with \#320 and \#800 abrasive paper 
amount of VGCF's. The heat treatment also reduces $<W_{s}$ $>$, whereas the dispersion of VGCF's more effectively reduces $<W_{s}>$ under the present experimental conditions. The abrasive particle size clearly influences $\mu$ and $<W_{s}$ $>$.

The relationships among the microhardness, the coefficient of friction, the specific wear rate and other mechanical properties are analyzed based on a simple model as follows: It is assumed that the tip of an abrasive particle indenting the material has a cross section area $A_{i}$ in the plane of undeformed material surface and a projection area $A_{s}$ on the plane perpendicular to the sliding direction. The shape factor $(g)$ is defined as

$$
g=\frac{A_{s}}{A_{i}}
$$

If the tip of the abrasive particle is a cone with an angle $\xi$ from the sliding direction, then $g$ is given by $(\tan \xi) / \pi$. It is assumed that the force $(P)$ in parallel to the sliding direction which is required to apply to the abrasive particle for sliding the particle is in proportion to $A_{s}$. That is

$$
P=\sigma_{s} A_{s}
$$

where $\sigma_{s}$ is a proportional constant representing the resistance against sliding. The force $(F)$ perpendicular to the material surface which is required to apply to the abrasive particle for making the indentation with an area of $A_{i}$ is given by

$$
F=\sigma_{i} A_{i}=k H A_{i}
$$

where $\sigma_{i}$ is a proportional constant representing the resistance against indentation. This parameter is in proportion to the microhardness $(H)$ with the proportional constant $(k)$ of 1.08 . From equations (6) to (8), the coefficient of friction $(\mu)$ is derived as

$$
\mu=\frac{P}{F}=\frac{g \sigma_{s}}{k H}
$$

This equation differs from the equation derived by Ernst et al. ${ }^{43)}$ in which $g$ appears as an additional term. It is assumed that the volume lost by wear $(V)$ is in proportion to the volume swept by the tip of the particle in sliding. That is

$$
V=f A_{s} L
$$

where $L$ is the sliding distance and $f$ is a proportional constant representing the probability that the material is removed or plastically deformed forming a scar per one sweep of the abrasive particle. The parameter $f$, which will be called fracture probability, is zero for a completely elastic material with sufficiently large strain at fracture and reaches unity as the material is getting weaker. From equations (8) and (10), the wear rate $\left(W_{s}\right)$ is given by
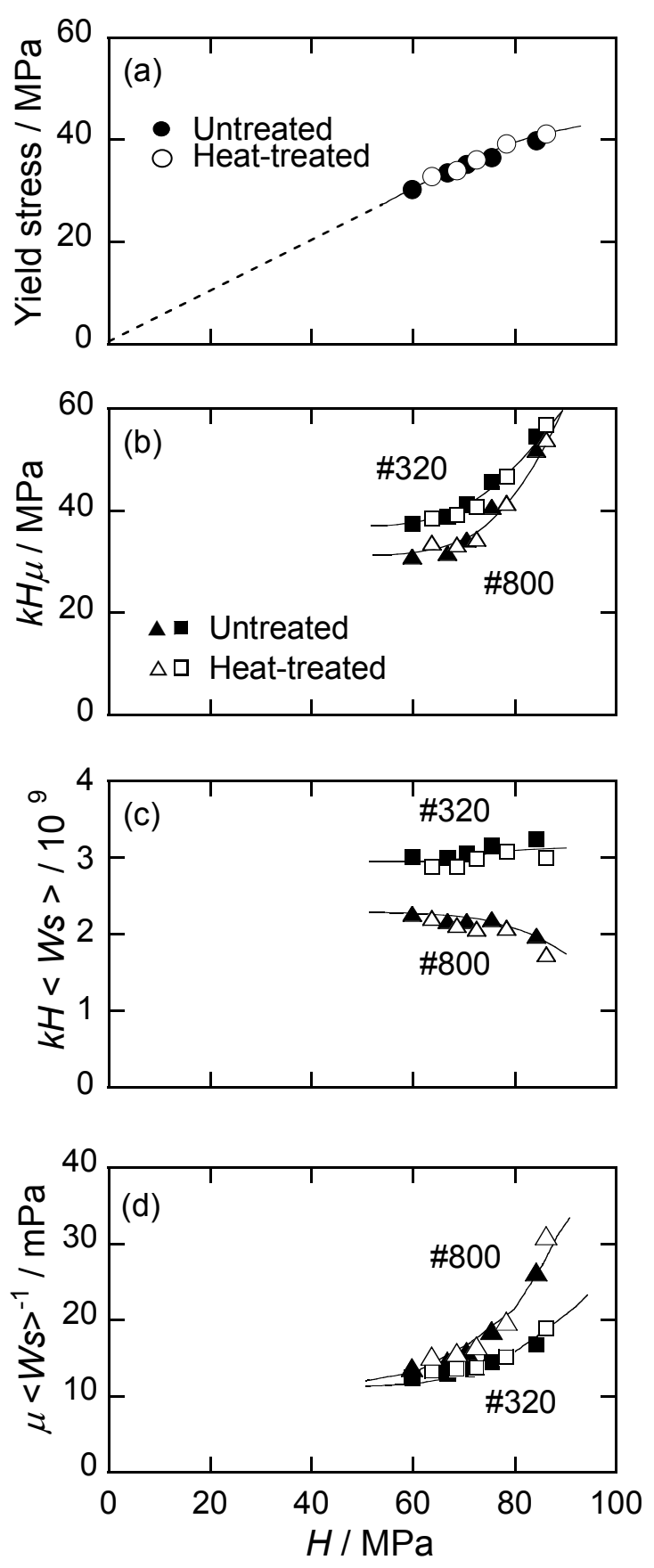

Fig. 11 (a) Tensile yield stress, (b) $k H \mu$ (c) $k H<$ $W_{s}>$ and (d) $\mu /<W_{s}>$ versus $H$ for untreated and heat-treated VGCF/PA6 composite fibers with various mass fractions of VGCF's. Fibers were worn with \#320 and \#800 abrasive paper

$$
W_{s}=\frac{V}{F L}=\frac{g f}{k H}
$$

A similar equation has already been derived ${ }^{44)}$ for a special case in which $f=1$ and the abrasive particle has a cone-shape tip. Equations (9) and (11) indicate that $\mu$ and 
$W_{s}$ are determined as a function of $H, \sigma_{s}, f$ and $g$. Conversely, relative variations in $\sigma_{s}$ and $f$ for a constant value of $g$ can be estimated using the equations.

$$
\begin{aligned}
& g \sigma_{s}=k H \mu \\
& g f=k H W_{s}
\end{aligned}
$$

The tensile yield stress of the composite fibers is plotted against $H$ in Fig. 11. The values of $k H \mu$ and $k H<$ $W_{s}>$ which correspond to the average values of $g \sigma_{s}$ and $g f$, respectively, are also shown in this figure.

It is expected that the tensile yield stress, $H$ and $\sigma_{s}$ are closely related to each other since all these parameters represent the resisting stress against deformation. As shown in Fig. 11(a), the tensile yield stress is actually in proportion to $H$ with a proportional constant of about 0.5 . The negative correlation between the tensile strength and $H$ known by comparing Figs. 7(c) and 11(a) is considered to be due to the fact that the tensile strength reflects the properties of the defects rather than the average fiber properties.

The dependence of $g \sigma_{s}$ on $H$ shown in Fig. 11(b) can be attributed mainly to the variation of the material parameter $\sigma_{s}$ since the dependence of the shape factor of the abrasive particle, $g$, on $H$ is considered to be small. It is known that the resistance $\sigma_{s}$ is an increasing function of $H$.

On the other hand, the dependence of $g \sigma_{s}$ on the abrasive particle size can be attributed mainly to the variation of $g$. Fig. 11(b) indicates that the difference of $g$ with the abrasive particle size is very small at large values of $H$ and about $20 \%$ at small values of $H$. If the tipof the abrasive particle has some special shape such as a cone, $g$ is independent of the indentation depth. With some other shapes, however, $g$ depends on the indentation depth. Therefore, even if the wear tests are carried out under the same contacting force, the indentation depth varies depending on the hardness of the material and the shape and the size of the abrasive particles. This causes the dependences of $g$ on $H$ and the abrasive particle size. This can be the reason for the variation of $g \sigma_{s}$ with the abrasive particle size at small values of $H$.

The dependences of $f g$ on $H$ and the abrasive particle size shown in Fig. 11(c) can be attributed mainly to the variation in $f$ since the variation of $g$ is small as shown above. The values of $f g$ is of the order of $10^{-3}$, and so is the fracture probability $f$. This means that permanent scars are produced after the material is swept with the abrasive particles 1000 times. The work given to the material in the sliding path of the abrasive particle, per unit volume and per one sweep of the abrasive particle, is equivalent to $\sigma_{s}$. Therefore, the total work given to the material in the sliding path until it is deformed, broken and eventually removed as wear debris, per unit volume is given by $\sigma_{s} / f$. Part of the work given to the material causes plastic deformation while some other part of the work is once stored elastically, turns into kinetic energy and eventually released as a heat. The material of unit volume is removed as wear debris when the total work given to the material reaches $\sigma_{s} / f$. Thus $\sigma_{s} / f$ is a measure of the toughness of the material against abrasive wear. The value of $\sigma_{s} / f$ can be obtained with the equation

$$
\frac{\sigma_{s}}{f}=\frac{\mu}{W_{s}}
$$

As shown in Fig. 11 (d), $\sigma_{s} / f$ increases with increasing $H$, indicating that the toughness against abrasive wear increases with increasing VGCF fraction. It is suggested that dispersion of VGCF's enhances the ability of the material to return to the undeformed shape after each sweep of the abrasive particles.

Fig. 11(d) shows that the toughness, $\sigma_{s} / f$, is smaller with the larger abrasive particle. This result can be interpreted similarly to the size effect in the strength. The strength of a material generally decreases with the increase in volume since the probability that the material contains defects increases. In the case of abrasive wear, the volume of the stressed region under each abrasive particle increases as the particle size increases and the fracture probability increases correspondingly.

The dependences of $\mu$ and $W_{s}$ on the VGCF fraction, the fiber structure and the abrasive particle size reflect the variations in $H, \sigma_{s}, f$ and $g$ so far shown. The values of
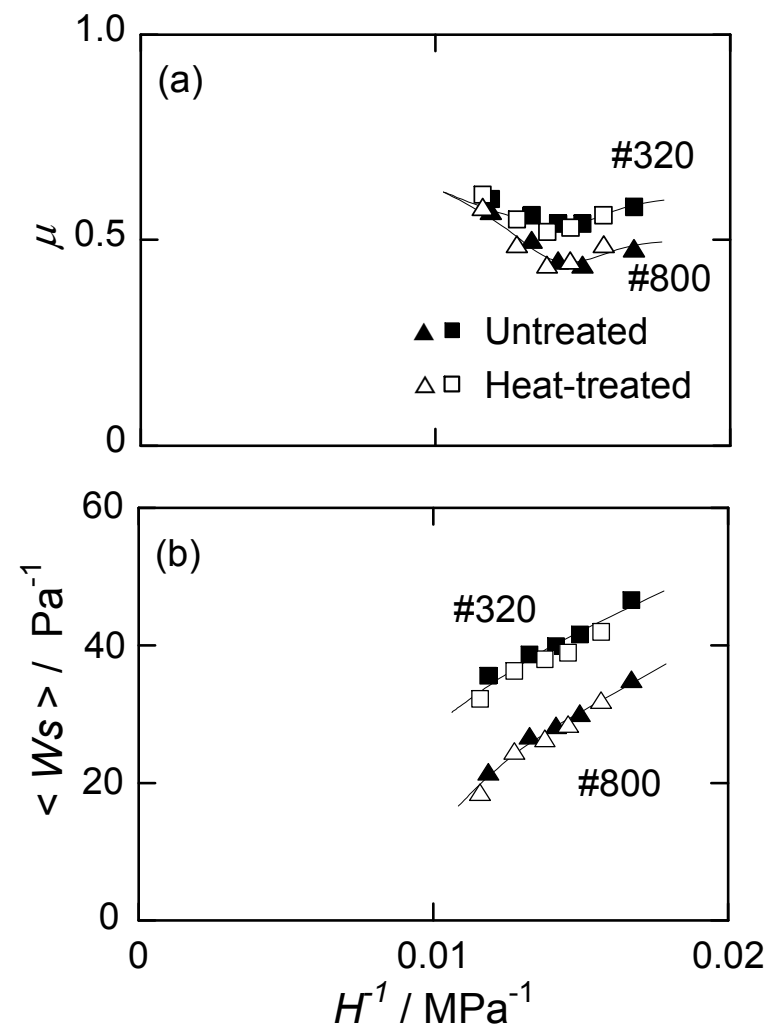

Fig. 12 (a) $\mu$ and (b) $<W_{s}>$ versus $1 / H$ for untreated and heat-treated VGCF/PA6 composite fibers with various mass fractions of VGCF's. Fibers were worn with \#320 and \#800 abrasive paper 
$\mu$ and $<W_{s}>$ are plotted against $1 / H$ in Fig. 12. If $\sigma_{s}$ is completely in proportion to $H$, then $\mu$ takes a constant value. The deviation of the relationship between $\sigma_{s}$ and $H$ from the proportionality produces the minimum of $\mu$ in Fig. 12(a). If $f$ is almost independent of the VGCF fraction but depends on the abrasive particle size, then $<$ $W_{s}>$ is in proportion to $1 / H$ with a slope depending on the abrasive particle size. This feature can be seen in Fig. 12(b).

\section{Conclusion}

The tribological properties under abrasive wear conditions, the tensile properties, the thermal properties and the structure have been investigated on the polyamide 6 (PA6) fibers dispersed with vapor grown carbon fibers (VGCF's) in order to analyze the effects of dispersing VGCF's on the wear resistance of PA6 fibers. The VGCF's worked as the nucleation agent to form the $\alpha$ phase during the melt spinning process. The crystallinity was increased by the heat treatment. With increasing VGCF fraction, the tensile modulus and the tensile yield stress increased while the tensile strength and the elongation at break decreased. The coefficient of friction $(\mu)$ and the wear rate $\left(W_{s}\right)$ could be related to the microhardness $(H)$, the resistance against sliding $\left(\sigma_{s}\right)$, the fracture probability $(f)$ and the shape factor $(g)$ as $\mu=$ $g \sigma_{s} / H$ and $W_{s}=g f / H$. The analysis based on these equations showed that for the composite fibers with varying VGCF fractions, tensile yield stress is in proportion to $H, \sigma_{s}$ is an increasing function of $H$ where its variation decreases as $H$ decreases, $f$ is roughly independent of $H$ but depends on the abrasive particle size and $g$ is roughly independent of $H$ and the abrasive particle size. Reflecting the variations of these parameters, the coefficient of friction decreased by dispersing a small amount of VGCF's but increased with increasing VGCF fraction at large VGCF fractions. The wear resistance increased with increasing VGCF fraction. Under the experimental conditions adopted in this study, the dispersion of VGCF's more effectively increased the wear resistance of PA6 fibers than the heat treatment.

\section{Acknowledgement}

The authors are deeply indebted to Professor emeritus Tsunamitsu Nakahara at Tokyo Institute of Technology for his useful suggestions and discussions.

\section{References}

[1] Tanaka, K., "Wear of Polymer-Based Composites," Sen'i Gakkaishi, 31, 8, 1975, 234-241. (in Japanese)

[2] Bekhet, N. E., "Tribological Behabior of Drawn Polypropylen," Wear, 236, 1999, 55-61.

[3] Kunugi, T., Chida, A. and Suzuki, A., "Preparation of High-Modulus Nylon 6 Fibers by Vibrating Hot
Drawing and Zone Annealing," Journal of Applied Polymer Science, 67, 1998, 1993-2000.

[4] Irisawa, T., Shioya, M., Kobayashi, H. and Kaneko, J., "Wear Resistance and Mechanical Properties of Polymeric Fibers Filled with Inorganic Fillers," Materials Research Society Symposium Proceedings, 977, 2006, 234-239.

[5] Irisawa, T., Kobayashi, H., Fujihira, K., Shioya, M. and Kaneko, J., "A Method to Determine Wear Rates of Fibers and Its Application to Polymeric Fibers Added with Inorganic Fillers," Wear, 268, 2010, 1148-1156.

[6] Ratner, S. B., Farberova, I. I., Radyukevich, O. V. and Lue'e, E. G., "Connection between Wear-Resistance of Plastics and Other Mechanical Properties," in James, D. I. (Ed.), Abrasion of Rubber," MacLaren and Sons Ltd., London, 1967, 145-154.

[7] Lancaster, J. K. "Abrasive Wear of Polymers," Wear, 14, 1969, 223-239.

[8] Lancaster, J. K. "Basic Mechanisms of Friction and Wear of Polymers," Plastics Polymer, 41, 1973, 297-306.

[9] Briscoe, B. J., Evans, P. D. and Lancaster, J. K., "Influence of Debris Inclusion on Abrasive Wear Relationships of PTFE," Wear, 124, 1988, 177-194.

[10] Tewari, U. S., Bijewe, J., Mathur, J. N. and Sharma, I., "Studies on Abrasive Wear of Carbon Fibre (Short) Reinforced Polyamide Composites," Tribology International, 25, 1992, 53-60.

[11] Bijwe, J., Awtabe, S., Satapathy, B. K. and Ghosh, A. K., "Influence of Concentration of Aramid Fabric on Abrasive Wear Performance of Polyethersulfone Composites," Tribology Letter, 17, 2004, 187-194.

[12] Lai, B., Alam, S. and Mathur, N., "Tribo-Investigation on PTFE Lubricated PEEK in Harsh Operating Conditions," Tribology Letter, 25, 2006, 71-77.

[13] Lhymn, C., "Effect of Normal Load on the Specific Wear Rate of Fibrous Composites," Wear, 120, 1987, 1-27.

[14] Petrovicova, E., Knight, R., Schdler, L. S. and Twardowski, T. E., "Nylon 11/Silica Nanocomposite Coatings Applied by the HVOF Process. 2. Mechanical and Barrier Properties," Journal of Applied Polymer Science, 78, 2003, 2272-2289.

[15] Bhimaraj, P., Burris, D. L., Action, J., Sawyer, W. G., Toney, C. G., Siegel, R. W. and Schadler, L. S., "Effect of Matrix Morphology on the Wear and Friction Behavior of Alumina Nanoparticle / Poly(ethylene)terephthalate Composites," Wear, 258, 2005, 1437-1443.

[16] Tong, J., Ma, Y. and Jiang, M., "Effects of the Wollastonite Fiber Modification on the Sliding Wear Behavior of the UHMWPE Composites," Wear, 255, 2003, 734-741. 
[17] Zhang, Z., Breidt, C., Chang, L. and Friedrich, K., "Wear of PEEK Composites Related to Their Mechanical Performances," Tribology International, 37, 2004, 271-277.

[18] Yanjiang, S., Peng, Z., Xiaodong, W. and Pei, H., "Effect of Filler Morphology on Tribological Performances of Polyimide Composites," Industrial Lubrication and Tribology, 62, 2010, 63-69.

[19] Chang, L., Zhang, Z., Zhang, H. and Friedrich, K., "Effect of Nanoparticles on the Tribological Behaviour of Short Carbon Fibre Reinforced Poly(etherimide) Composites," Tribology International, 38, 2005, 966-973.

[20] Zhang, H., Zhang, H. and Friedrich, K., "Effect of Fiber Length on the Wear Resistance of Short Carbon Fiber Reinforced Epoxy Composites," Composites Science and Technology, 67, 2007, 222-230.

[21] Xu, H., Feng, Z., Chen, J. and Zhou, H., "Tribological Behavior of the Polyamide Composite Coating Filled with Different Fillers under Dry Sliding," Journal of Applied Polymer Science, 104, 2007, 2554-2560.

[22] Suresha, B., Chandramohan, G., Jawali, N. D. and Siddaramaiah, "Effect of Short Glass Fiber Content on Three-Body Abrasive Wear Behaviour of Polyurethane Composites," Journal of Composite Materials, 41, 2007, 2701-2713.

[23] Breuer, O. and Sundararaj, U., "Big Returns from Small Fibers: A Review of Polymer/Carbon Nanotube Composites," Polymer Composites, 25, 2004, 630-645.

[24] Liu, T., Phang, I. Y., Shen, L., Chow, S. Y. and Zhang, W. D., "Morphology and Mechanical Properties of Multiwalled Carbon Nanotubes Reinforced Nylon-6 Composites," Macromolecules, 37, 2004, 7214-7222.

[25] Andrews, R. and Weisenberger, M. C., "Carbon Nanotube Polymer Composites," Current Opinion in Solid State and Materials Science, 8, 2004, 31-37.

[26] Kobayashi, H., Shioya, M., Tanaka, T., Irisawa, T., Sakurai, S. and Yamamoto, K., "A Comparative Study of Fracture Behavior between Carbon Black/Poly(Ethylene Terephthalate) and Multiwalled Carbon Nanotube/Poly(Ethylene Terephthalate) Composite Films," Journal of Applied Polymer Science, 106, 2007, 152-160.

[27] Chai, H., Yan, F. and Xue, Q., "Investigation of Tribological Properties of Polyimide/Carbon Nanotube Nanocomposites," Materials Science and Engneering A, 364, 2004, 94-100.

[28] Enomoto, K., Yasuhara, T. and Ohtake, N., "Frictional Properties of Carbon Nanofiber Reinforced Polymer Matrix Composites," New Diamond and Frontier Carbon Technology, 14, 2004, 11-20.
[29] Kanagaraj, S., Mathew, M. T., Fonseca, A. and Rocha, L. A., "Tribological Characterisation of Carbon Nanotubes/Ultrahigh Molecular Weight Polyethylene Composites, The Effect of Sliding Distance," International Journal of Surface Science and Engineering, 4, 2010, 305-321.

[30] Meng, H., Sui, G. X., Xie, G. Y. and Yang, R., "Friction and Wear Behavior of Carbon Nanotubes Reinforced Polyamide 6 Composites under Dry Sliding and Water Lubricated Condition," Composites Science and Technology, 69, 2009, 606-611.

[31] Li, J., Fang, Z., Tong, L., Gu, A. and Liu, F., "Polymorphism of Nylon-6 in Multiwalled Carbon Nanotubes/Nylon-6 Composites," Journal of Polymer Science B, 44, 2006, 1499-1512.

[32] Fornes, T. D. and Paul, D. R., "Crystallization Behavior of Nylon 6 Nanocomposites," Polymer, 44, 2003, 3945-3961.

[33] Friedrich, K., Zhang, Z. and Schlarb, A. K., "Effects of Various Fillers on the Sliding Wear of Polymer Composites," Composites Science and Technology, 65, 2005, 2329-2343.

[34] Bijwe, J., Tewari, U. S. and Vasudevan, P., "Friction and Wear Studies of Bulk Polyetherimide," Journal of Materials Science, 25, 1990, 548-556.

[35] Friedrich, K., Kocsis, J. K. and Lu, Z., "Effects of Steel Counterface Roughness and Temperature on the Friction and Wear of PE(E)K Composites under Dry Sliding Conditions," Wear, 148, 1991, 235-247.

[36] Roselman, J. C. and Tabor, D., "The Friction and Wear of Individual Carbon Fibres," Journal of Physics D, 10, 1977, 1181-1194.

[37] Barrioz, J. C., Mazuyer, D., Kapsa, P., Chateauminois, A. and Bouquerel, F., "On the Mechanisms of Abrasive Wear of Polyamide Fibres," Wear, 255, 2003, 751-757.

[38] Ramesh, C. and Gowd, E. B., "High-Temperature X-Ray Diffraction Studies on the Crystalline Transitions in the $\alpha$ - and $\gamma$ - Forms of Nylon-6," Macromolecules, 34, 2001, 3308-3313.

[39] Shibaya, M., Tsuji, Y., Sakurai, S., Ishihara, H. and Yoshihara, N., "Structure Variations of High Tenacity Nylon 6 Fiber on Cyclic Temperature Changes," Journal of the Textile Engineering, 52, 2006, 107-112. (in Japanese)

[40] Giza, E., Ito, H., kikutani, T. and Okui, N., "Fiber Structure Formation in High-Speed Melt Spinning of Polyamide 6/clay Hybrid Nanocomposite," Journal of Macromolecular Science, Part B, 39, 2000, 545-559.

[41] Yoshioka, Y. and Tashiro, K., "Structural Change in the Brill Transition of Nylon m/n (1) Nylon 10/10 and its Model Compounds," Polymer, 44, 2003, 7007-7019. 
[42] Morishima, M., Morikawa, A., Gocho, H., Jojima, E. and Shimizu, Y., "Mechanical Property of Nylon 6 Fiber with Various Diameters in Standard, Wet and Water-Immersed Conditions," Sen'i Gakkaishi, 58, 2002, 294-298. (in Japanese)
[43] Ernst, H. and Merchant, M. E., "Surface Friction of Clean Metals," Proceeding Special Summer Conference on Friction and Surface Finish, 1940, 76-101.

[44] Rabinowicz, E. and Mutis, A., "Effect of Abrasive Particle Size on Wear," Wear, 8, 1965, 381-390. 